\title{
Real Time Monitoring in Romania: Part of the Bigger Picture
}

\author{
Keetie Roelen*
}

\begin{abstract}
In 2009-10, a Rapid Assessment of the Social and Poverty Impacts of the Economic Crisis was undertaken in Romania to gain timely and in-depth information about the impact of the crisis on particular groups in society. This article assesses the use of a rapid assessment as a Real Time Monitoring (RTM) tool and more broadly discusses the value of RTM in a middle-country context with a myriad of existing data collection and monitoring systems. Findings suggest that a rapid assessment can prove a cost-efficient and valuable tool if the appropriate research expertise and experience is available. The assessment of micro- and macro-level challenges in the education, health and child protection sectors points towards the importance of RTM initiatives being embedded within the wider data collection and monitoring systems. The translation of data and reports into policy influence and impact emerged as a recurrent theme in this study.
\end{abstract}

\section{Introduction}

Romania is a middle-income country with a wellestablished landscape of data collection and monitoring efforts. Sectors including education, health and others operate their own monitoring information and reporting systems. The National Institute of Statistics (Institutul National de Statistica, INS) is a key player in collecting sectoral and survey-based data and the provider of official statistics in Romania. The economic crisis in 2008 inspired a particular data collection exercise that aimed to gain more timely and in-depth information about the impact of the crisis on particular groups in society. A Rapid Assessment of the Social and Poverty Impacts of the Economic Crisis in Romania (hereafter referred to as RA) was undertaken in 2009 and 2010. The aim of this article is two-fold; firstly it aims to assess the process and impact of the Rapid Assessment of the Economic Crisis as a Real Time Monitoring (RTM) tool, and secondly to assess the potential for RTM in a middleincome country and EU Member State context, with strong routine data collection efforts already in place. This is done with a particular focus on the most vulnerable groups in Romania, and most specifically focused on children.

The RA was assessed as part of a larger study on Real Time Monitoring for the Most Vulnerable (RTMMV) undertaken by IDS and UNICEF in
2012. Romania was identified as a front-runner country in the CEE/CIS region and the RA in Romania as a particular RTM initiative for closer consideration. The assessment of the RA as an RTM tool and the potential for RTM within Romania's wider data collection monitoring landscape was largely informed by primary qualitative data collection and assessment of secondary information including the RA reports and documentation on Romania's wider monitoring and evaluation efforts. Fieldwork took place in February 2012 and included semistructured interviews with key informants, semistructured focus group discussions (FGDs) and a stakeholder workshop. Participants included a wide range of stakeholders involved in the RA and the overall monitoring practice in Romania, particularly from education, health and child protection sectors and included UNICEF staff, local researchers, government counterparts, district and local-level policymakers, NGO representatives and participants of the RA. Qualitative data collection took place in Bucharest, Sibiu and Alba Iulia.

The structure of this article is as follows: firstly, the process of the RA is described, after which both the process and outcomes are assessed against the criteria of quality of information, inclusivity, relevance to potential users and costs and sustainability. Secondly, the micro- and 
macro-level challenges in Romania's wider data collection and monitoring landscape are discussed on the basis of observations in the sectors of education, health and child protection. Finally, the potential of RTM, and the RA as a particular RTM tool, in the context of Romania is discussed and concluding remarks are provided.

\section{Rapid Assessment (RA) of the economic crisis}

The RA in Romania 2009-10 was initiated in 2009 and took place over a two-year period in 2009 and 2010. The RA was initiated by the World Bank and UNICEF in 2009 after the first signs of the adverse impact of the global economic crisis on life in Romania. The RA consisted of four rounds of data collection across a two-year time period in 2009-10. While the first round contained both quantitative and qualitative research elements, the latter three rounds focused on qualitative data collection only. Four reports were published following the RA data collection and analysis; three focused on the findings of the first three separate rounds (Stanculescu and Marin 2009, 2010; Stanculescu and Grigoras 2009), while the fourth presented a combined analysis of all four rounds (UNICEF 2011).

Although the RA was initiated on the premise of it being a study assessing the transmission of the economic crisis through two mediating channels, namely the labour market and remittances, the focus gradually shifted towards assessing the impact on children and vulnerable groups. This shift in focus can largely be attributed to institutional shifts with respect to those involved in the RA; the World Bank discontinued its engagement after the first round. With UNICEF solely responsible for continuing the RA, the exercise gradually shifted focus. Following recognition after the second round that the original research framework lacked a child perspective and did not provide enough information about the impact on the most vulnerable, the sample was expanded to include areas with particularly vulnerable groups (including Roma communities). To enhance the focus on children even further, the fourth and final round extended the qualitative research by adding retrospective questions in order to gain an understanding of how the economic crisis impacted care and living conditions for children.

The performance of the RA as an RTM tool is assessed against the criteria of the quality of information, its inclusivity, relevance to potential users and costs and sustainability.

\subsection{Quality of information}

Quality of information of the RA in Romania was considered to be of a high standard. The qualitative panel study was implemented across all four rounds by the same local research institute. The long-standing experience of the research institute and the expertise of its researchers in terms of data collection and analysis in Romania positively contributed to the quality of findings. In addition, the fact that the same institute, and researchers within that institute, were involved throughout the whole process ensured consistency in both data collection and analytical methods. At the same time, the institute showed flexibility in adapting the sampling frame and fieldwork guides to accommodate the shifting focus of the RA from round one to four to consider the impacts of the economic crisis on the most vulnerable and children. Any adjustments that were made, including the expansion of the sample and addition of retrospective questions, were undertaken with due care in order not to undermine previous findings or their quality.

Although the decision to maintain the qualitative component only across all four rounds was agreed upon by all partners involved in the process of the $\mathrm{RA}$, there was also recognition that having both quantitative and qualitative information to feed into the RA would have strengthened the quality of information and concurrent possibilities for dissemination and policy influence. Although the four rounds of qualitative data allowed for important insights and improved understandings, there was a feeling that they lacked the power to make more general statements about the impact of the economic crisis. Most respondents agreed that future initiatives should seek to include both quantitative and qualitative components. Such a quantitative component could be based on sentinel sites rather than being nationally representative to ensure feasibility and sustainability of a survey over time.

\subsection{Inclusivity}

As the RA initially focused on capturing information about how the economic crisis was transmitted to the household level through labour markets and remittances, the sample for the qualitative sentinel survey focused on public 
and private sector workers and those receiving remittances. After increased recognition that vulnerable groups and children were not adequately included, the sample was expanded to capture experiences of Roma groups. Despite the expansion of the sample to include Roma households in round three, and many of the respondent households having children, the study did not explicitly tease out findings for children at large, or vulnerable children in particular. The focus on children, and the effect of the economic crisis on them, was only really introduced in the third round, following changes of the stakeholders involved. Given the socioeconomic status of Roma groups, findings for these households and their children may provide some indication of the effects of the crisis on a particular group of vulnerable children. However, the study did not explicitly sample other groups of vulnerable children, such as those living outside of parental care, disabled children or children in poor households.

The set and focus of questions was expanded across rounds to learn more about how household members' experienced the impact of the economic crisis and how they felt it impacted upon their children. In the fourth round, questions were added on job security, employment of household members, household income, access to credit and coping strategies. The combination of these questions and their answers over time provided insight into micro-level impacts at the household level. Recall bias is an obvious concern when adding such a retrospective component to the research, which was addressed by asking for main events that are easier to recall or remember (such as job loss, asking relatives for support, taking children out of extracurricular activities) rather than detailed information about amount of money earned or borrowed.

RA participants themselves largely considered the inclusion and participation in the exercise to have been a positive experience. FGD participants in Sibiu as well as key informants that were interviewed as part of the RA indicated that they felt their voices were being heard and that it was a rare opportunity for others to come and learn from their experiences. An NGO respondent also indicated that participation in the RA helped to reflect on the NGO's situation and the community more widely in a critical and constructive manner, particularly as the RA was conducted over an extended period of time at regular intervals. Participants of FGDs in the Roma community in Alba Iulia were less convinced that participation in such discussions or research into the impact of the economic crisis would lead to any tangible changes in policy or outcomes. None of the participants had seen the end result of the RA, and would have liked to know more about the results and publication of findings.

\subsection{Relevance to potential users}

The interviews with key informants at both central and local level, and across different sectors and services, clearly indicated that awareness about the RA and its findings was limited beyond those having been directly involved. Many respondents had never heard of the exercise and had not seen its final report. It was clear that the RA lacked a coherent and wellconsidered dissemination strategy that would ensure the findings from the RA would become known to a wide audience, and that they would influence thinking about what to do in the event of a future crisis. A few reasons can be identified as to why such a strategy appeared to be missing.

Firstly, the many changes in stakeholders involved in this RA, including the departure of those having initiated the exercise, and the concurrent shifts in focus of the RA complicated the identification of an overarching objective of the RA and its target audience. In addition, these institutional shifts themselves are likely to have undermined the potential policy impact of the RA. The different perspectives from which to assess the impact of the economic crisis are likely to appeal to different audiences (i.e. from the Ministry of Economics and Finance and the Ministry of Labour, Family and Social Protection to the Ministry of Education and the General Direction of Child Protection within the Ministry of Labour, Family and Social Protection), and have different implications for how findings can be used for policy impact. Different ministries and policymakers will have different requirements for information, and the organisations providing the information and research (i.e. the World Bank and UNICEF) may use different channels through which to seek policy influence.

A second reason for the lack of a dissemination strategy appears to be the emphasis on the process of the RA rather than on the concurrent outcomes and what to do with them. Questions 
such as 'What should this RA and its findings achieve?' and 'Who should be reached and benefit from this RA?' were not considered explicitly throughout the process of the RA, and therefore not translated into a coherent and consistent dissemination strategy. Although reports at the end of each round of the RA were sent out to relevant government counterparts and ministries, there was no deliberate consideration as to how these reports could influence those counterparts. The final report of the RA has not been officially launched. In terms of feedback to participants and a wider public audience, the publication of an easy-to-read version of the report was considered. Time and budget constraints prevented such a report and its dissemination from materialising.

\subsection{Costs and sustainability}

High-quality quantitative and qualitative data collection requires time and human resources, and can quickly become very costly. The longitudinal nature of this RA adds to these costs as it requires investments over time on a regular basis. A nationally representative quantitative survey as carried out in the first round of this RA will prove too costly in many contexts. A large sample is required, and panel surveys need strong mechanisms to track respondents over time to keep the attrition rate to a minimum. The use of sentinel sites is an option to contain costs, while a deliberate sampling strategy can ensure that findings allow for drawing conclusions more broadly.

An important distinction in reference to costs and sustainability of the RA as an RTM tool is that between the process of data collection and reporting and the process of in-depth analysis. While data collection and reporting processes can be automated to a certain level, and thereby be undertaken at fairly low cost once systems are in place, data analysis requires more time, human capacity and often clearance at multiple levels before public release. This presents a prudent trade-off between more detailed and in-depth information on the one hand and costeffectiveness and timeliness of information on the other hand. The time period between data collection and actual reporting, including an in-depth analysis for this RA, was between one and two months. This is a fairly short turnaround time, making an analysis of the impact of the economic crisis as real-time as possible. The extent to which such a quick turnaround is possible will depend greatly on the expertise and experience of the researchers involved and the quality of research tools.

\section{Data collection and monitoring in Romania: the bigger picture}

The analysis of a particular RTM tool in a middleincome country also allows for consideration of the 'bigger picture' question of the potential of RTM initiatives in a context with abundant data collection and monitoring systems already in place. The value of doing so is two-fold: on the one hand it extends the discussion of the value of RTM beyond developing countries, and on the other hand it allows for drawing important lessons for the development and use of RTM in contexts where few data collection and monitoring systems are yet in place but rapidly emerging.

Indicative assessments of monitoring systems in the education, health and child protection sectors pointed towards a number of micro- and macrochallenges. Micro-level challenges are those challenges that pertain to issues within particular monitoring systems and tools and compromise the resulting data in terms of accuracy, depth of information and timeliness, while macro-level challenges refer to wider policy-related issues, pertaining to coordination and cooperation, and use of data for policy change.

\subsection{Micro-level challenges}

A first challenge that became particularly evident in the sectors of education and child protection is the lack of adequate in-house expertise to meaningfully and critically process the data collected. This capacity does not refer to the technical ability to deal with systems of data collection but rather with the statistical skills that are required to check and verify the data that is collected at municipal or county level. The health sector appeared to have a real advantage over the other two sectors in terms of data collection and processing as the National Centre for Health Statistics has dedicated statisticians that work with the data collected through their county level Directorates on a day-to-day basis. The absence of such statistical capacity in the education and child protection sector slows down processes of data verification and 'confrontation' and may compromise data quality and accuracy.

Secondly, all sectors struggle with getting those with the information to feed this into the data monitoring system. This includes doctors in the 
health sector, teachers or school directors in the education sector and case workers in terms of child protection. Against the backdrop of budget cuts and a general squeeze on resources in the public sector, the time available to those providers of information is becoming scarcer, making this a particularly pertinent challenge. Although the pressure and administration created by filling forms is widely recognised by those responsible for collating and reporting the data at county and national level, various mechanisms are now being considered to increase the response rate. Both the education and child protection sector are exploring options for making funding conditional upon full, accurate and timely provision of data. There was also a widespread sense that those filling the forms and providing the data should be more aware of what the data is used for, and why its provision is important - not only at national level but also for data providers themselves. Rather than meeting the requirements of data provision, people should use the data to their own advantage.

Thirdly, differences between definitions underlying the calculation of particular indicators and coding schemes used to denote particular services or localities were mentioned across all sectors. Such differences occur between different bodies involved in the data collection and reporting process, and particularly between data collection efforts by the ministerial bodies and the processing and reporting efforts by INS. Although such differences pertain to the nittygritty details of the data collection and reporting process, they can have far-reaching implications. It can result in considerable discrepancies between outcomes, and also requires considerable time to check and verify data for harmonisation purposes. The lack of consistency causes tensions and frustration at both ends of the process.

Finally, problems with respect to the absence of adequate IT capacity were particularly apparent in the education and child protection sectors. In both cases, no in-house capacity was available for trouble-shooting at county or national level or to adapt the system so that it reflects current legislation. As a result, child protection case workers have to work with software that asks for data that are no longer relevant or that does not allow for the adequate information to be entered, undermining consistency of data across the country. With respect to the education sector, the monitoring system is currently administered by the IT company rather than the Ministry, which raises issues around public data in the hands of the private domain.

\subsection{Macro-level challenges}

Firstly, it was widely recognised, and also observed during the visit, that there is a lack of coordination and knowledge both within and across sectors about data collected and available. This limited level of coordination results in duplication of efforts but also in discrepancies and inconsistencies across the data collected. In addition, it hampers cooperation between different bodies involved in the data collection and reporting process, both horizontally and vertically. Those having to provide the information (at the level of schools and hospitals, for example) are reluctant to do so in the absence of strong coordination and with lack of knowledge about the use of data. Those collecting and compiling the data at both the sub-national and national level feel equally less inclined to collaborate with each other without sectoral harmonisation of efforts.

Secondly, in terms of data use and policy influence, it became clear that data collection and monitoring for many is a case of 'ticking the boxes' rather than a realisation that such data is to be used to improve programmes and policies. Although the limited use of data towards 'evidence-based policymaking' can in part be attributed to a lack of (statistical) capacity in the various line ministries as outlined above, it also signifies the absence of vision and priority-setting in terms of the use of data. The example of poverty assessments and the lack thereof on a regular basis is a case in point. The National Institute of Statistics (Institutul National de Statistica, INS) collects high-quality data on a regular and timely basis through a variety of surveys, including the Household Budget Survey (HBS), which holds all information required for regular poverty assessments. However, the INS has no mandate for analysing this data. With respect to poverty assessments, the responsibility sits with the Ministry of Labour, Family and Social Protection, but they do not have the capacity for undertaking such an analysis and to produce regular reports on the situation with respect to poverty in Romania. Although INS calculates poverty figures following Eurostat guidelines and based on national guidelines, such information is not contextualised, analysed or disseminated in 
regular reports or otherwise. As such, it can be said that the wealth of the HBS data remains under-utilised.

This challenge is not exclusive to one particular ministry but holds across all sectors and for the majority of line ministries; staff are either not available or do not have the technical capacity to process data and undertake critical analysis. The lack of such capacity has largely been attributed to a lack of financial resources. Resource allocation is very much dependent on priorities by politicians and policymakers, and the systematic shortage of investment in statistical capacity across the board signifies a clear lack of vision in terms of use of data and importance of monitoring for policy improvement. In 2009, legislation was put in place stipulating that statisticians or statistical capacity should be put in place in all bodies of public administration to process and verify data before sending it to INS. Such in-house capacity could lead to great efficiency gains as there is less need for data 'confrontation' and verification. Data quality would be increased at the same time. Nevertheless, the development of such in-house capacity has not yet materialised in the majority of ministries. Although budget and capacity constraints play an important role, it has to be noted that the lack of political will is an equally important factor. This may hold especially true in terms of politically sensitive issues, such as poverty or child protection.

\section{Discussion and conclusion}

The purpose of this article was two-fold: on the one hand to assess the performance of the Rapid Assessment of the Social and Poverty Impacts of the Economic Crisis in Romania as an RTM tool in and of itself, and on the other hand to assess the potential of RTM within the broader data collection and monitoring landscape in Romania. This was done with a particular angle on the most vulnerable groups in Romania, and most specifically focused on children.

Overall, the quality of the RA in Romania was found to be good, making RAs an attractive option for assessing the impact of a crisis on a particular group in society in a timely manner. The RA was undertaken with the continuous involvement of an experienced and highly skilled institute that ensured quality of the qualitative data collection and analysis was maintained across the four rounds. It also strengthened the qualitative data collection process as trust was built over time between interviewers and respondents in both KIIs and FGDs. A particular focus on children and the most vulnerable, both in terms of sampling and questions, was not introduced until the third round of the RA following the shifts in involvement of stakeholders, but the RA managed to tease out micro-level effects and reduce recall bias by asking questions in relation to key life events. Participants of the interviews and discussions were generally positive about the experience, indicating that they saw this as a rare opportunity to make their voices heard.

Despite the overall positive experience, the RA also faced challenges across the different stages of setup and implementation. The evolving focus of the study across the four rounds compromised its consistency and clarity about the message that the reports were to convey. Also, although the decision to maintain the qualitative component only was widely agreed upon, there was also recognition that having both quantitative and qualitative information to feed into the RA would have strengthened the outcomes and concurrent possibilities for dissemination and policy influence. Although the four rounds of qualitative data allowed for important insights and improved understandings, there was a feeling that they lacked the power to make more general statements about the impact of the economic crisis. The most pertinent challenges with respect to the RA in Romania refer to dissemination and research uptake. There was no clear dissemination or policy impact plan from the outset, leading to little policy uptake as a result. This lack of influence was further compounded by the changing involvement of partners and political volatility in Romania, making it hard to explicitly link RA reports to programme or budget cycles to maximise impact.

An assessment of data monitoring systems in the education, health and child protection sectors identified a number of micro- and macro-level challenges in terms of data collection and reporting both within and across those sectors. Micro-level challenges include the lack of in-house statistical capacity, low response rates, discrepancies between definitions and coding schemes and lack of technical capacity. Macrolevel challenges pertain to issues concerning the system in which separate monitoring initiatives 
take place. A lack of coordination and knowledge both within and across sectors about the data that is collected and available results in the duplication of efforts, as well as discrepancies and inconsistencies in the data collected by different sectors. In addition, it hampers cooperation between different bodies involved in the data collection and reporting process, both horizontally and vertically. In terms of data use and policy influence, it became clear that data collection and monitoring for many is a case of 'ticking the boxes' rather than a realisation that such data is to be used to improve programmes and policies. Although the limited use of data towards 'evidence-based policymaking' can in part be attributed to a lack of (statistical) capacity in the various line ministries as outlined above, it also signifies the absence of vision and priority-setting in terms of the use of data. More technical constraints pertain to differences between definitions underlying the calculation of particular indicators and coding schemes used to denote particular services or localities as well as lack of technical capacity to work with, maintain and update management information systems. Such lack of consistency and capacity causes tensions and frustration at both ends of the process; those supplying and those demanding the data.

The assessment of the RA as well as the data monitoring systems in education, health and child protection to consider the potential role of RTM within those systems, gives rise to two larger questions that have to be kept in mind in further development and rollout of RTM initiatives, particularly in contexts where routine data collection and monitoring systems are not yet in place.

\section{Notes}

* This documentation would not have been possible without the support and input from UNICEF Romania, UNICEF CEE/CIS Regional Office and UNICEF HQ. In

\section{References}

Stanculescu, M.S. and Grigoras, V. (2009) Rapid Assessment of the Social and Poverty Impacts of the Economic Crisis in Romania, June 2009, Bucharest: UNICEF and World Bank Stanculescu, M.S. and Marin, M. (2010) Rapid Assessments of the Social and Poverty Impacts of the Economic Crisis in Romania, Round 3, July 2010, Bucharest: UNICEF and World Bank
Firstly, it is clear that any monitoring tool or initiative will not be developed or used in isolation; rather they are (or will be) part of a bigger system. For RTM to be developed and used in a sustainable manner in the future, the recognition that any initiative does not stand on its own and needs to be embedded in wider data collection, monitoring and reporting systems is imperative. Systems-thinking is required not only within sectors to link new data collection and monitoring efforts with existing ones, but also across sectors.

Secondly, how to make research and studies work for policy impact was a recurring theme in the assessment of this RA and an issue that many involved in data collection and analysis are grappling with in Romania. The RA and its problems in terms of dissemination and policy impact serve as an illustration of wider concerns about how to translate research into policy impact. The politically volatile and organisationally fluid situation in Romania was considered a major impediment in 'evidencebased' policymaking. Politicians and policymakers' decisions were considered to be driven by other, more politically charged, motives rather than evidence about who and where the most vulnerable are, and what could or should be done to reach them most effectively and appropriately. In addition, the unpredictable political situation causes counterparts to change frequently, and for them to also change their minds about the course of action or appropriate policies. Many concerns were raised about how to 'make noise' in such a volatile situation, and how to make sure that the noise is reaching the appropriate audience.

addition, the author is greatly indebted to the team of researchers of CERME, who provided technical and logistical support throughout the process of this country case study.

Stanculescu, M.S. and Marin, M. (2009) Rapid Assessments of the Social and Poverty Impacts of the Economic Crisis in Romania, Round 2, November 2009, Bucharest: UNICEF and World Bank UNICEF (2011) Impacts of the International Economic Crisis in Romania 2009-2010, Bucharest: UNICEF 